\title{
Efectividad del programa educativo "Cuidando con amor" en los conocimientos, actitudes y prácticas sobre prevención de úlceras por presión de los cuidadores de pacientes adultos mayores del Hospital San Isidro Labrador - EsSalud, Lima, Perú
}

\author{
Effectiveness of the educational program "Caring With Love" in knowledge, attitudes and practices \\ on the prevention of pressure ulcers in elderly caregivers of patients at the San Isidro Labrador \\ Hospital - EsSalud, Lima, Peru
}

Orfelina Arpasi Quispe ${ }^{1}$

\begin{abstract}
RESUMEN
Objetivo: Determinar la efectividad del programa educativo "Cuidando con amor" en los conocimientos, actitudes y prácticas sobre prevención de úlceras por presión de los cuidadores de pacientes adultos mayores del Hospital San Isidro Labrador - EsSalud. Material y Métodos: El estudio fue de diseño preexperimental, de tipo cuantitativo con un solo grupo a quien se aplicó una preprueba y posprueba. Se utilizó el muestreo no probabilístico, a conveniencia del investigador, basado en criterios de inclusión y exclusión previamente establecidos, la muestra estuvo conformada por 30 cuidadores. El programa estuvo organizado en 15 sesiones educativas teórico-prácticas con una frecuencia de 2 veces por semana durante 4 meses. Para medir los conocimientos y actitudes se aplicó un cuestionario y para la práctica se utilizó una guía de observación elaborados por la autora. Resultados: Los resultados obtenidos en cuanto al nivel de conocimiento sobre prevención de UPP antes del programa fueron: $46.7 \%$ muy malo, $36.7 \%$ malo y $23.3 \%$ regular, observándose un incremento al $100 \%$ excelente, después del programa. Las actitudes de los cuidadores antes del programa fueron favorables en un $96.7 \%$ y después del programa llegaron a ser $100 \%$ favorables. En cuanto al nivel de práctica antes del programa se encontró un $86.7 \%$ no favorable y después del programa $100 \%$ favorable. La prueba $\mathrm{t}$ - student indica que existe diferencia significativa de 0.00 , entre el nivel de conocimientos, actitudes y prácticas de prevención de UPP antes y después del programa educativo. Conclusiones: El programa "Cuidando con amor" fue efectivo en el incremento del nivel de conocimientos, actitudes y prácticas sobre prevención de úlceras por presión en los cuidadores de adultos mayores del Hospital II Clínica Geriátrica San Isidro Labrador.
\end{abstract}

Palabras clave: Conocimientos, actitudes, prácticas, cuidador, adulto mayor, úlcera por presión.

\section{SUMMARY}

Objective: To determine the effectiveness of the educational program "Caring With Love" in knowledge, attitudes and practices on the prevention of pressure ulcers in elderly caregivers of patients at the Hospital San Isidro Labrador - EsSalud. Material and Methods: The study was pre experimental design, quantitative with one group to whom a pre-test and post-test was applied. Non-probability sampling, the convenience of the investigator, based on criteria previously established inclusion and exclusion was used, the sample consisted of 30 caregivers. The program was organized 15 educational sessions in theory and practice with a frequency of 2 times a week for 4 months. To measure knowledge and attitudes questionnaire was applied and practice elevorados an observation guide was used by the author. Results: The results obtained in the level of knowledge about prevention of pressure ulcers before the program were: $46.7 \%$ very bad, bad $36.7 \%$ and $23.3 \%$ regularly, with an increase to $100 \%$ excellent, after the program. The attitudes of caregivers before the program were favorable in $96.7 \%$ and after the program became $100 \%$ favorable. Regarding the level

\footnotetext{
${ }^{1}$ Jefa del Departamento de Enfermería, Clínica San Isidro Labrador EsSalud. Docente de la Facultad de Ciencias de la Salud, Universidad Peruana Unión, Lima. Perú.
} 
of practice before the program encotró $86.7 \%$ unfavorable and after the program $100 \%$ favorable. The test $\mathrm{t}$ - student indicates significant difference of 0.00 between the level of knowledge, attitudes and practices of prevention of pressure ulcers before and after the educational program. Conclusions: The "Caring With Love" was effective in increasing the level of knowledge, attitudes and practices on the prevention of pressure ulcers in elderly caregivers Hospital Geriatric Clinic II San Isidro Labrador.

Keywords: Knowledge, attitudes, practices, caregiver, elderly, pressure ulcer.

\section{INTRODUCCIÓN}

Ante los cambios demográficos y epidemiológicos que se vienen produciendo a nivel mundial y de manera particular en América Latina y el Caribe, es indispensable la preparación para responder a las demandas de la creciente población adulta mayor que representa un $12 \%$ de la población mundial, según la OMS (2009).

El envejecimiento humano es un fenómeno universal inevitable y de gran trascendencia debido a las repercusiones sanitarias, económicas y sociales. Según reportes a nivel mundial, la población adulta mayor está aumentando considerablemente debido al incremento de la expectativa de vida, disminución de tasas de natalidad y mortalidad, entre otros factores.

Según el acuerdo de Kiev la OMS (1979) considera adultos mayores a los de más de 60 años para los que viven en los países en vías de desarrollo y de 65 años a los que viven en países desarrollados. La OPS (1999), ajustó la edad de 65 y más para considerarlo como adulto mayor. Para hacer el análisis de la población por estructura de edad en el Perú el Ministerio de Salud (2011) presenta el programa de atención integral de salud donde considera al adulto mayor desde los 65 años a más.

En el Perú la población adulta mayor representa el $9,1 \%$ del total de la población, siendo la proyección estimada del 12.5\% para el año 2025 (INEI, 2007). Ante esta situación, los requerimientos de los servicios sanitarios también se ven incrementados, tal como lo demuestran las estadísticas del Seguro Social de Salud (EsSalud), institución peruana que ha experimentado un progresivo aumento en la atención de las personas adultas mayores, llegando a un $13.2 \%$ del total de atenciones, un $26.4 \%$ de los egresos hospitalarios a nivel nacional y en $32.3 \%$ del total de egresos en Lima (EsSalud, 2006).

En relación a las causas de hospitalización en adultos mayores, la primera causa son las infecciones: neumonía, infección del tracto urinario y sepsis y accidente cerebrovascular (Varela, 2000 y Ortiz 2001). Estos diagnósticos médicos están fuertemente asociados al desarrollo de úlceras por presión en el adulto mayor, por el impacto que tienen estas dos enfermedades en el estado general y en la movilidad de este grupo etáreo.

Solís (2011), menciona que las úlceras por presión son uno de los síndromes geriátricos que ocasionan mayor inversión socioeconómica y sanitaria. Los efectos de las úlceras por presión radican en una estancia hospitalaria cinco veces mayor que en pacientes sin esta condición, un incremento en la mortalidad, un aumento significativo de complicaciones infecciosas y comorbilidad, y un incremento en los costos de manejo, estimándose en un costo de 489 dólares por úlcera, con un tiempo medio de tratamiento de 116 días (INEI, 2007 y Chigne, 2000).

Este problema no es exclusivo de las personas adultas mayores porque puede suceder a cualquier edad, pero los adultos mayores son los que están en mayor riesgo debido a la inmovilización por la declinación biológica normal, la mayor morbilidad, la mayor susceptibilidad a enfermarse, especialmente, por patologías de resolución retardada que reducen aún más el margen de defensa y autonomía (Varela, 2009). Sumado a esto, Venegas (2010), refiere que aunque una persona de cualquier edad desarrolla úlceras por presión si no cambia de posición con frecuencia, en los adultos mayores incrementan este riesgo por cambios de la piel relacionados a la edad, incluyendo pérdida de humedad, de grasa subcutánea e incremento en la fragilidad.

De acuerdo con Montero (2007), las úlceras por presión constituyen un serio problema que afecta principalmente a las personas que permanecen en cama durante largos periodos de tiempo, como consecuencia de traumas o enfermedades; afecta la capacidad funcional del adulto mayor, aumentando considerablemente la morbimortalidad de las personas afectadas (Masías, 2001).

En la actualidad a nivel mundial, se encuentran pocos registros sobre la problemática de las úlceras por presión y la prevención de las mismas. Según Cañón (1998), estudios realizados en Europa, Sudáfrica y Canadá permiten establecer que la 
Efectividad del programa educativo "Cuidando con amor" en los conocimientos, actitudes y prácticas sobre prevención de úlceras por presión de los cuidadores de pacientes adultos mayores del Hospital San Isidro Labrador - EsSalud, Lima, Perú

prevalencia de las personas con úlceras por presión en el medio hospitalario puede llegar hasta un $45 \%$ en los enfermos crónicos y por encima del $9 \%$ de todas las personas hospitalizadas, constituyéndose en un problema de salud no solo en personas en estados crónicos sino también en personas en estados agudos.

Según Flemming (2008), en el Reino Unido, las úlceras por presión se registraron de un $5 \%$ a $32 \%$ de pacientes ingresados en un hospital general. Así mismo, Carrasco (2002), refiere que en Brasil el 2\% de las úlceras por presión son muy severas y pueden comprometer la vida del enfermo. Se calcula que su incidencia en la población general es del 1.7\% entre los 55 y 69 años y del $3.3 \%$ entre los 70 y 75 años. El $60 \%$ aproximadamente se desarrollan en el hospital y más del $70 \%$ ocurren en mayores de 70 años. Lo más importante es que el 95\% de las úlceras por presión son evitables y por ello es prioritaria la prevención.

En un trabajo realizado por Gallo (2008), en el Perú en el Programa de Atención Domiciliaria (PADOMI) - EsSalud reporta que el 53,3\% de ancianos se encontraba en un rango de 80-89 años de edad, de los cuales el 43,4\% presentó un riesgo medio de desarrollar úlceras por presión, teniendo en cuenta las condiciones determinantes que lo incrementan.

En el Hospital II Clínica Geriátrica San Isidro Labrador (HIICGSIL) de la Red Asistencial Almenara (RAA) de EsSalud, según el informe emitido por el comité de calidad (2008), los síndromes o problemas geriátricos con mayor incidencia fueron: úlceras por presión, estreñimiento, incontinencia urinaria y fecal, inmovilización, caídas y trastornos del sueño. Durante el 2011, se hospitalizaron pacientes adultos mayores con pluripatologías con alto índice de susceptibilidad a presentar síndromes geriátricos especialmente las úlceras por presión, pues el $40 \%$ de los pacientes que ingresan al hospital vienen con úlceras por presión secundarias. Al preguntar a los cuidadores o familiares las razones de la presencia de las lesiones o úlceras por presión, las respuestas son "no sé cómo se formó", "nadie me enseñó a evitarlo", o simplemente "no hay quien cuide a mi paciente". Además, la aplicación de la escala de Norton por las enfermeras indica que el $80 \%$ del total de pacientes que ingresan al hospital tienen riesgo a desarrollar úlceras por presión (Oficina de Estadística CGSIL, 2011).

Estas cifras son preocupantes, pues las úlceras por presión son prevenibles en un $95 \%$, por lo que es necesario disponer de estrategias de educación y prevención en programas de capacitación en salud enfocados a las personas cuidadoras, siendo en la mayoría de los casos uno o varios miembros de la familia, para que ellas obtengan herramientas que beneficien al usuario o usuaria (Venegas, 2010).

Según la OMS (2009), la familia es la unidad de Salud que incluye en el núcleo familiar a todas las personas que viven bajo una misma unidad residencial entre los cuales existen lazos de dependencia, obligaciones recíprocas y que por lo general, pero no siempre, están ligados por lazos de parentesco. La familia asume un rol protagónico como agente de cuidado de los adultos mayores, de manera particular en la prevención de úlceras por presión cuando el paciente tiene una permanencia prolongada en cama.

Gallo (2008), refiere que el 55,6\% de los ancianos son cuidados por sus hijos y otros familiares, presentando en ambos casos un riesgo medio para desarrollar úlceras por presión. Situación que alerta a los profesionales de salud y principalmente a las enfermeras, quienes deben implementar programas de educación continua y entrenamiento supervisando al familiar y/o cuidador, para prevenir el desarrollo de úlceras por presión en esta población. En España, el cuidado principal de los ancianos residentes en domicilio recae el cuidado, en el $46 \%$ de los casos, en los hijos. En el Perú el INEI (2007), reporta que el $35 \%$ de los ancianos de 60 años a más viven con los hijos. El agente de cuidado o proveedor de cuidados tiene que aprender a participar en el cuidado de su paciente y hacerlo de modo continuo, lo que constituye una característica humana fundamental. Los requisitos infaltables, para realizar dicho cuidado, son el aprendizaje y el uso de conocimientos para llevar a cabo las acciones que faciliten la prevención eficaz y oportuna de las úlceras por presión (Orem, 1980).

En ese sentido, Gallo (2008), refiere que "lamentablemente en nuestro medio no existen programas preventivo-promocionales a favor del adulto mayor, falta mayor incentivo en las instituciones de formación de profesionales de salud para interesarse en el paciente geriátrico, así como de un adiestramiento adecuado para su atención en los diferentes niveles de salud".

Ante la necesidad del cuidado de este tipo de pacientes en el hogar, posterior al alta, se implementó en la Clínica Geriátrica San Isidro Labrador, desde al año 2009, el programa familiar acompañante conducido por el profesional de Enfermería que tiene por objetivo capacitar a los familiares $o$ cuidadores en el cuidado del adulto mayor en el hogar. El programa consta de 12 sesiones de aprendizaje: Higiene de manos, alimentación por sonda nasogástrica o por vía oral, administración de medicamentos, baño en cama, control de glicemia, control de funciones vitales, movilización, cuidado 
espiritual, entre otros. Sin embargo, se ha observado que los familiares de los pacientes preguntan constantemente sobre la prevención y tratamiento de úlceras por presión, refiriendo además en el caso de presentar úlceras por presión: "no me lo llevo a mi paciente hasta que estén curadas las heridas".

De allí la importancia de que todas las personas encargadas del cuidado de los adultos mayores deberían capacitarse tempranamente en la prevención y manejo de los principales problemas geriátricos, como el estreñimiento, la incontinencia urinaria y/o fecal, las caídas, las úlceras por presión, entre otras.

El trabajo de investigación presenta el programa "Cuidando con amor", una propuesta de capacitación para los cuidadores de los adultos mayores en la prevención de úlceras por presión, que surge debido a la necesidad de aprendizaje del cuidador del paciente adulto mayor de la Clínica Geriátrica San Isidro Labrador de la Red Asistencial Almenara, teniendo como objetivo mejorar sus conocimientos, actitudes y prácticas para la prevención de las úlceras por presión de los pacientes adultos mayores.

\section{MATERIAL Y MÉTODOS}

El diseño del estudio fue preexperimental con un solo grupo a quien se aplicó preprueba y posprueba a fin de determinar la efectividad del programa. Se utilizó el muestreo no probabilístico, a conveniencia del investigador, basado en criterios de inclusión y exclusión previamente establecidos. La muestra estuvo conformado por 30 cuidadores de los pacientes Adultos Mayores hospitalizados en el HIICGSIL en el mes de marzo del 2012. El programa estuvo organizado en 15 sesiones educativas teórica prácticas con una frecuencia de 2 veces por semana durante 4 meses.

Para medir conocimientos se aplicó un cuestionario de selección múltiple; para actitudes se utilizó la escala de Likert y para la práctica se aplicó guía de observación. Para la validez de los instrumentos, se empleó el juicio de expertos en el área. Asimismo, con la finalidad de comprobar la confiabilidad de los instrumentos fueron sometidos a una prueba de campo. Se utilizó la prueba del coeficiente del Alpha de Crombach, obteniendo un coeficiente de Alpha de Crombach alta; en la variable conocimiento sobre prevención de úlceras por presión fue de 0,72 ; en la variable actitud de 0,89 y en las prácticas de 0.71 , determinando que existe un buen nivel de confiabilidad, es decir una buena relación entre los ítems indicados.

El análisis de los datos fue procesado con un programa estadístico SPSS 20, 2011. Los estadísticos que se utilizaron fueron: estadísticos descriptivos, tablas de frecuencias en particular la t-student para muestras relacionadas.

\section{RESULTADOS}

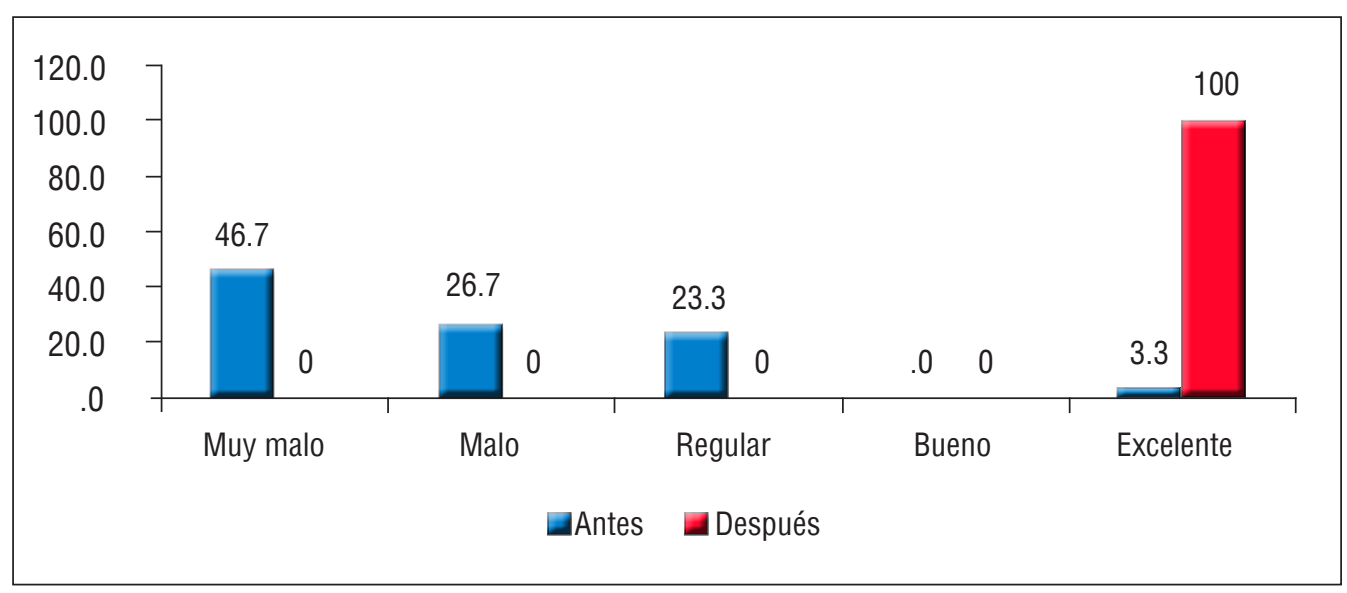

Figura 1

Nivel de conocimiento de los cuidadores sobre la prevención de UPP antes y después de la aplicación del programa "Cuidando con amor". 
Efectividad del programa educativo "Cuidando con amor" en los conocimientos, actitudes y prácticas sobre prevención de úlceras por presión de los cuidadores de pacientes adultos mayores del Hospital San Isidro Labrador - EsSalud, Lima, Perú

En la figura 1 se observa que antes de aplicar el programa "cuidando con amor", el $46.7 \%$ de la muestra presentó un nivel de conocimiento muy malo sobre medidas preventivas de UPP, el $50 \%$ presentó un nivel de conocimiento entre malo y regular y solo un $3.3 \%$ presentó un nivel de conocimiento excelente. Después de aplicar el programa, se observó que el $100 \%$ tuvo un nivel de conocimientos excelente.

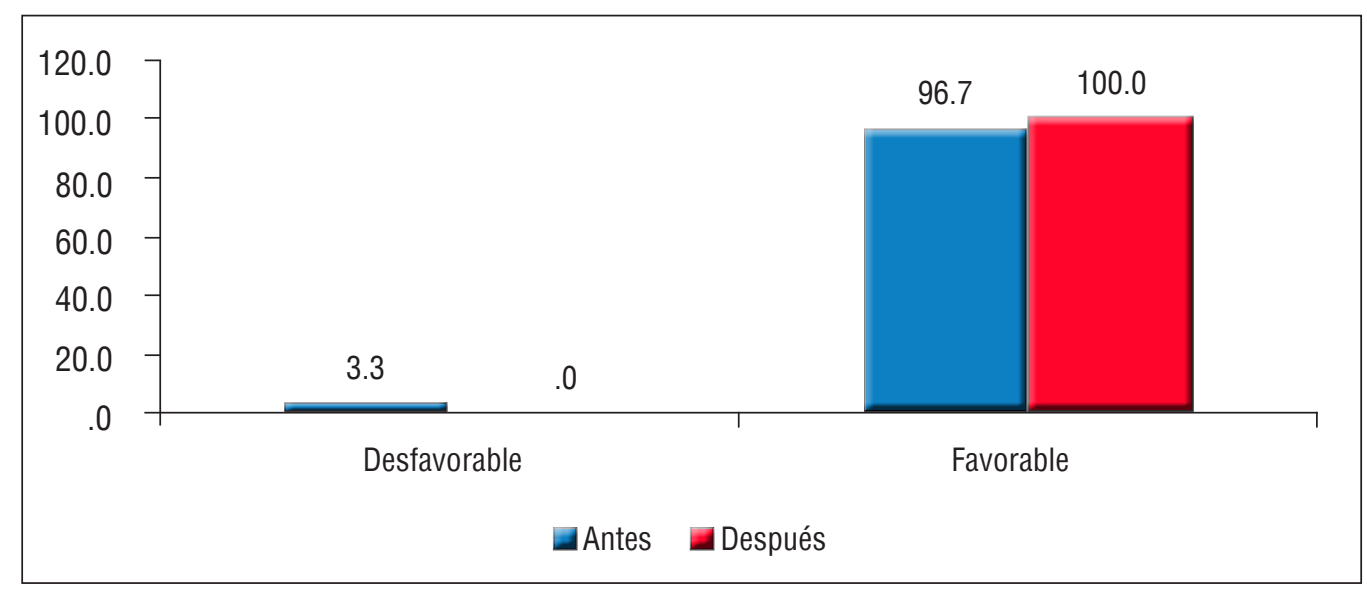

Figura 2

Actitud de los cuidadores hacia las medidas preventivas de la UPP antes y después de la aplicación del programa "Cuidando con amor".

En la figura 2 muestra que antes de aplicar el programa, el $96.7 \%$ presentó actitud favorable hacia las medidas preventivas de las UPP y el $3.3 \%$ presentó actitud desfavorable. Después de aplicar el programa, el $100 \%$ presentó actitud favorable.

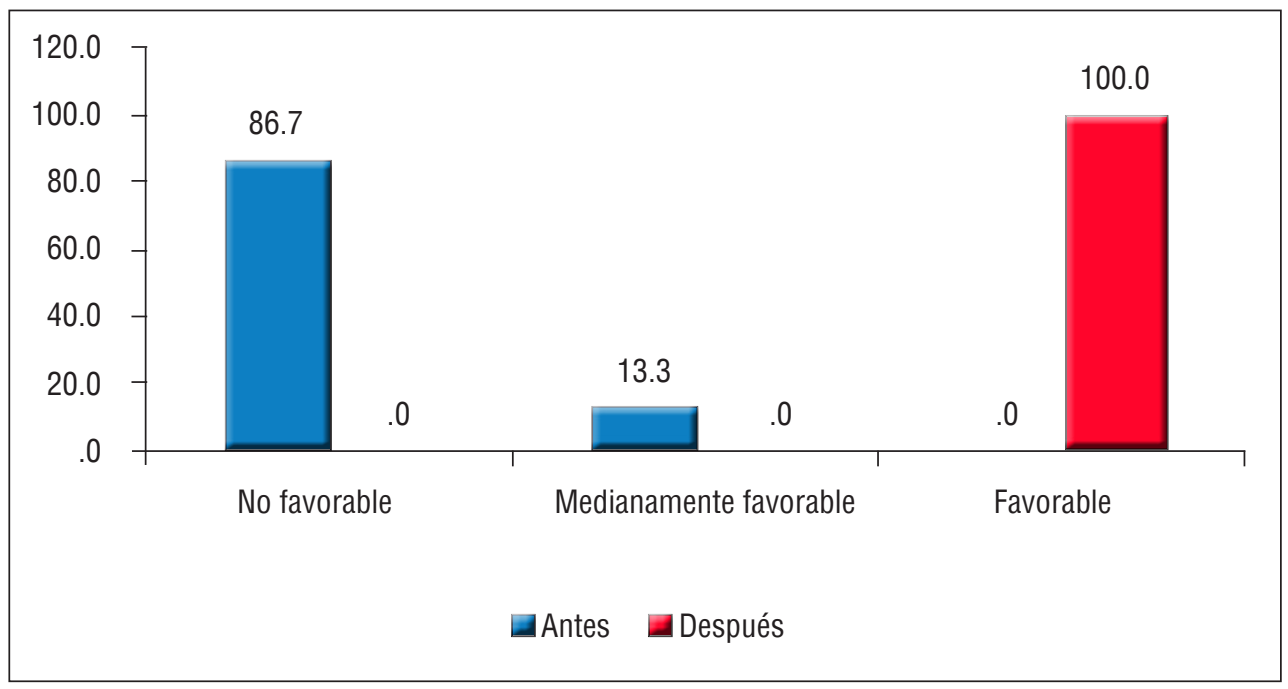

Figura 3

Práctica de los cuidadores sobre las medidas preventivas de la UPP antes y después de la aplicación del programa "Cuidando con amor".

La figura 3 muestra que antes de aplicar el programa, el $86.7 \%$ evidenció una práctica no favorable de las medidas preventivas de UPP y el $13.3 \%$ presentó una práctica medianamente favorable. Después de aplicar el programa el 100\% de los cuidadores evidenció una práctica favorable. 
Tabla 1

Análisis de comparación de medias para muestras relacionadas con el nivel de conocimiento, actitudes y prácticas antes y después de la aplicación del programa "Cuidando con amor".

\begin{tabular}{lllll}
\hline Criterios & Media & N & Desviación tip. & Error \\
\hline Conocimiento - Antes & 7,73 & 30 & 4,291 & 0,783 \\
Conocimiento - Después & 19,80 & 30 & 0,610 & 0,111 \\
Actitudes - Antes & 38,50 & 30 & 6,437 & 1,175 \\
Actitudes - Después & 50,00 & 30 & 0,000 & 0,000 \\
Práctica - Antes & 4,00 & 30 & 2,729 & 0,498 \\
Práctica - Después & 19,47 & 30 & 0,900 & 0,164 \\
\hline
\end{tabular}

En la tabla 1, para determinar la efectividad del programa "Cuidando con amor" en el nivel de conocimiento, actitud y práctica de los cuidadores sobre prevención de úlceras por presión, se utilizó el análisis estadístico y comparación de medias para muestras relacionadas. En el nivel de conocimiento se obtuvo una media de 7.73 puntos, antes de la aplicación del programa, después de este se obtuvo una media de 19,18 puntos. La respuesta obtenida en la actitud fue una media de 38.5 puntos, antes de la aplicación del programa y después de este se obtuvo una media de 50 puntos. En la práctica la respuesta fue una media de 4.00 puntos antes de la aplicación del programa, después de este se obtuvo una media de 19.47 puntos. Concluyendo así que el programa "Cuidando con amor" fue efectivo en el nivel de conocimiento, actitud y práctica de los intervenidos incrementando sus conocimientos, actitud y práctica en la prevención de las úlceras por presión.

Tabla 2

Prueba t de muestras relacionadas del nivel de conocimiento, actitudes y prácticas antes y después de la aplicación del programa "Cuidando con amor".

\begin{tabular}{|c|c|c|c|c|c|c|c|c|}
\hline \multirow{3}{*}{ Puntaje } & \multicolumn{5}{|c|}{ Diferencias relacionadas } & \multirow[b]{3}{*}{$\mathrm{t}$} & \multirow[b]{3}{*}{ gl } & \multirow{3}{*}{ 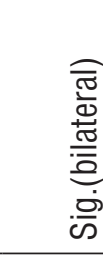 } \\
\hline & \multirow[t]{2}{*}{ Media } & \multirow[t]{2}{*}{$\begin{array}{l}\text { Desviación } \\
\text { tip. }\end{array}$} & \multirow[t]{2}{*}{$\begin{array}{l}\text { Error } \\
\text { tip. de la } \\
\text { media }\end{array}$} & \multicolumn{2}{|c|}{$\begin{array}{l}95 \% \text { intervalo de } \\
\text { confianza para la } \\
\text { diferencia }\end{array}$} & & & \\
\hline & & & & Inferior & Superior & & & \\
\hline $\begin{array}{l}\text { Conocimiento } \\
\text { antes y después }\end{array}$ & $-12,067$ & 4,315 & ,788 & $-13,678$ & $-10,456$ & $-15,318$ & 29 & 0,000 \\
\hline $\begin{array}{l}\text { Actitudes antes y } \\
\text { después }\end{array}$ & $-11,50$ & 6,437 & 1,175 & $-13,904$ & $-9,096$ & $-9,79$ & 29 & 0,000 \\
\hline $\begin{array}{l}\text { Práctica antes y } \\
\text { después }\end{array}$ & $-15,467$ & 2,556 & ,467 & $-16,421$ & $-14,512$ & $-33,143$ & 29 & 0,000 \\
\hline
\end{tabular}

En la tabla 2, para determinar la efectividad del programa "Cuidando con amor" en el nivel de conocimiento, actitud y práctica sobre la prevención de úlceras por presión de los cuidadores, se utilizó el análisis estadístico de prueba t-student para muestras relacionadas, teniendo como respuesta para el nivel de conocimiento una diferencia promedio de 12.067 puntos del antes sobre el después de la aplicación del programa; además, se puede determinar la efectividad del programa por el análisis del Sig. $=0,000$ que es menor que el valor de prueba $(\mathrm{p}=0,05)$. Para la actitud de los cuidadores la respuesta obtenida fue de una diferencia promedio de 11.50 puntos del antes sobre el después de la aplicación del programa; además, se puede determinar la efectividad del programa por el análisis del Sig.= 0,000 que es menor que el valor de prueba $(\mathrm{p}=0,05)$. La respuesta obtenida para la práctica fue una diferencia promedio de 15,467 
Efectividad del programa educativo "Cuidando con amor" en los conocimientos, actitudes y prácticas sobre prevención de úlceras por presión de los cuidadores de pacientes adultos mayores del Hospital San Isidro Labrador - EsSalud, Lima, Perú

puntos del antes sobre el después de la aplicación del programa. Además, se puede determinar la efectividad del programa por el análisis del Sig.= 0,000 que es menor que el valor de prueba $(\mathrm{p}=0,05)$. Por lo que se concluye la aceptación de la hipótesis alterna, confirmando la efectividad del programa "Cuidando con amor" en el nivel de conocimiento, actitud y práctica.

\section{DISCUSIÓN}

Las úlceras por presión constituyen un importante problema de salud pública, por sus repercusiones en el nivel de salud de quienes las padecen, la calidad de vida de los usuarios y su entorno y el consumo de recursos para el sistema de salud. Lo más resaltante es que el $95 \%$ de las úlceras por presión pueden prevenirse (Hibbs P. 1987, Waterlow J. 1996) citado por el Servicio Andaluz de Salud (2004) por lo que es necesario disponer de estrategias de educación a través de programas de capacitación en salud enfocados a las personas cuidadoras de adultos mayores para que ellas obtengan herramientas que se trasmitan y beneficien al usuario.

La familia es la principal proveedora de cuidados de salud, y que el cuidado se basa en relaciones afectivas y de parentesco (García, Mateo \& Maroto, 2004). Además, Armas (2008), refiere que la familia cumple un rol importante en los cuidados a tener en la prevención de úlceras por presión en sus pacientes, siendo tres las dimensiones básicas: la higiene, movilización y alimentación, ya que constituye el mejor apoyo para la recuperación rápida del paciente, así como también el de prevenir las posibles complicaciones en el paciente con permanencia prolongada en cama.

Bajo este marco, se diseñó el programa educativo "Cuidando con amor" contando con la participación de 30 cuidadores, de los cuales el 100\% tenían una relación de parentesco familiar con el paciente adulto mayor. El $60 \%$ fueron mujeres, la edad del $73 \%$ oscilaba entre 35 y 65 años, el $60 \%$ tenían un grado de instrucción entre primaria y secundaria y un $40 \%$, instrucción superior. El $57 \%$ presentaban un nivel socioeconómico medio y el $43.3 \%$ bajo y el $52 \%$ tenía como principal ocupación ama de casa.

Datos similares respecto al género han sido reportados por Rivera, Dávila y Gonzáles (2011), en el estudio "sobrecarga en cuidadores de pacientes con demencia tipo Alzheimer" donde el 82,7\% de los cuidadores eran mujeres, cifra cercana al 84.1\% encontrado por Crespo y López (2008). Lo anterior concuerda con la mayoría de los autores, quienes refieren que la responsabilidad de cuidar es fundamentalmente femenina. (García, Mateo \& Maroto, 2004). Las mujeres no solo asumen de forma mayoritaria el papel de cuidadoras principales, también se responsabilizan de tareas más pesadas y demandantes, y dedican más tiempo a cuidar que los hombres, lo que genera una clara inequidad de género con el consiguiente aumento del riesgo de sobrecarga de la cuidadora.

En el programa cuidando con amor, los cuidadores participantes tenían bajo su cuidado a adultos mayores con riesgo de desarrollar úlceras por presión. Al realizarse la captación para su participación el $100 \%$ mostró interés en aprender cómo evitar este problema, refiriendo que tenían conocimientos escasos sobre cómo prevenir las úlceras por presión, lo cual se evidenció en los resultados antes del programa "Cuidando con Amor", donde el 46.7\% de los cuidadores participantes presentaron un nivel de conocimiento sobre prevención de úlceras por presión muy malo, el $36.7 \%$ un nivel malo y $23.3 \%$ un nivel regular. Resultados que difieren de los encontrados por Armas (2008), en familiares de pacientes con permanencia prolongada en cama, en donde el 58\% presentaron un nivel de conocimiento medio, el 22\% un nivel bajo y el 10\% un nivel alto.

Los conocimientos en el programa "Cuidando con amor" que menos puntaje obtuvieron fueron los relacionados a la localización frecuente de las úlceras por presión, con un $16,7 \%$ de respuestas correctas, en segundo lugar los conocimientos de los factores de riesgo: la higiene y la movilización con un $30 \%$ y $33 \%$ de respuestas correctas respectivamente, datos que se observan en el apéndice I.

La úlcera por presión se debe a la interrupción del flujo sanguíneo, que se produce en zonas sometidas a presión y con la participación de otros factores como fricción, humedad y contaminación local con orina o heces (Salgado, 2002) y (Chávez, 2000). Se estima que el $18 \%$ de las personas mayores de 65 años tienen problemas para movilizarse sin ayuda y que a partir de los 75 años la cifra sube al 50\%, situación que aumenta la vulnerabilidad y riesgo de producir UPP (Capuñay, 2000).

El rol de la familia en el cuidado se orienta a la prevención de UPP, a través de la valoración de la piel que debe estar enfocada hacia las prominencias óseas, ya que estas son los sitios más frecuentes para el desarrollo de úlceras, de ahí que debe observarse la integridad y coloración de la piel en la búsqueda de zonas isquémicas; además, está dirigido a disminuir el grado de presión y/o rozamiento, ello puede obtenerse mediante la utilización de técnicas de cambios de posición y la elección de una adecuada superficie de 
apoyo que mitigue al máximo la presión. Además la realización de ejercicios pasivos y masajes, que contribuya a disminuir la aparición de UPP (Armas, 2008).

Según Pancorbo (2007), el éxito de la prevención está en función del conocimiento, las habilidades y las actitudes de las mismas. Armas (2008), refiere que los aspectos relacionados al conocimiento están dados en primer lugar por la dimensión de movilización, es decir en la frecuencia medida en el tiempo de los cambios de posición y ejercicios pasivos y a la técnica correcta de los mismos; seguido de la dimensión de higiene referido a la observación de la piel, coloración, integridad y la identificación de las zonas isquémicas. Gallo (2008), afirma que la prevención de las úlceras por presión comienza con la identificación y control de los factores de riesgo, seguido de la inspección diaria de la piel y finaliza con la adopción de una serie de medidas específicas dirigidas a evitar la aparición de las úlceras por presión. Las úlceras por presión ocurren en pacientes frágiles con pluripatologías y estado general deficiente, si a ello se suma las posibles complicaciones de la lesión local.

Diversos estudios han demostrado que existe la necesidad de la intervención de un programa educativo en la prevención de UPP a fin de mejorar los conocimientos. Según Flores, De La Cruz, Ortega y Hernández (2010), es necesario dar a conocer a los cuidadores la razón de los cambios frecuentes de posición, estimular la circulación de la piel que cubre las prominencias óseas, cambiar de posición al usuario cuando menos cada dos horas, según sus necesidades. Todas estas prácticas de prevención deben ser del conocimiento de las personas cuidadoras, ya que las úlceras por presión se pueden prevenir si se cumple con los cuidados básicos, por lo tanto el conocimiento de estos es importante.

Al respecto, en el programa "Cuidando con amor" se diseñaron 15 sesiones educativas y talleres prácticos de prevención de úlceras por presión dirigida a los familiares cuidadores, donde se enfatizó en los factores de riesgo de desarrollar úlceras por presión, cambios posturales y movilización, examen diario, higiene y dispositivos que se usan para prevenir las UPP y alimentación saludable. Además, se fortaleció el aprendizaje en la prevención de la aparición de nuevas úlceras por presión.

Después del programa "Cuidando con Amor", el nivel de conocimientos de los cuidadores se incrementó al 100\% en el nivel excelente.

Según la UNESCO (1997), la educación de adultos debe orientarse a las necesidades básicas de aprendizaje, es decir, a lo que la persona necesita para desarrollarse en el ámbito personal, familiar, laboral y social.

Por otro lado, el MINSA (2005), refiere que la educación de adultos está llamada a ser muy eficaz, eficiente y efectiva. Para que esto sea posible se requiere hacer uso de una adecuada metodología, que, partiendo de la identificación de las necesidades de capacitación, y luego de una adecuada planificación y una cuidadosa ejecución, logre obtener el impacto deseado. Para obtener impacto se requiere que exista una concordancia entre los objetivos de la capacitación y la metodología empleada, en la cual los contenidos educativos y las experiencias educativas se diseñen en forma coherente con los objetivos educativos y las características de la población a la que está destinada la capacitación.

Teniendo en cuenta que los conocimientos, actitudes y prácticas están estrechamente relacionadas entre sí, se desarrolló la capacitación a los familiares cuidadores a través del programa "Cuidando con amor". Las sesiones educativas tuvieron una hora de duración, se contó con el apoyo de equipos multimedia, se elaboró un módulo educativo para adultos, el mismo que fue distribuido a cada participante; la metodología empleada fue eminentemente participativa diseñada para adultos y/o adultos mayores. Otro aspecto a destacar fue la oferta de horarios flexibles para los participantes al programa, se programó las sesiones educativas tres veces por semana en diferentes turnos ya sea mañana o tarde.

Se puede concluir que los resultados del nivel de conocimientos excelente del $100 \%$ de los participantes sobre prevención de úlceras por presión, evidencia la efectividad del programa "Cuidando con amor", porque los familiares cuidadores pasaron de un nivel de conocimientos muy malo y malo en su mayoría a un nivel de conocimientos excelente, así como lo confirma la prueba estadística de $\mathrm{t}$ - student, con un nivel de significancia de 0.00 (Ver tabla 2).

Si bien es cierto los conocimientos son un primer eslabón para la práctica de conductas generadoras de salud, es necesario que la persona se sienta predispuesta favorablemente a aceptar el conocimiento que llevará a la acción. En ese sentido, Arrivillaga, Salazar y Correa (2003), sostienen que el factor cognitivo determina o predispone los cambios conductuales y la adopción de estilos de vida saludables, sin embargo, por sí solo no es un indicador de cambio conductual. Se ha demostrado que cierta cantidad de información es necesaria para iniciar el proceso que conducirá a un cambio de comportamiento. 
Efectividad del programa educativo "Cuidando con amor" en los conocimientos, actitudes y prácticas sobre prevención de úlceras por presión de los cuidadores de pacientes adultos mayores del Hospital San Isidro Labrador - EsSalud, Lima, Perú

El cambio conductual es un proceso dinámico y una parte normal de la vida, un modo que tienen las personas de crecer, desarrollarse y adaptarse, es frecuente que el cambio tarde en ser aceptado, sobre todo si las actitudes de las personas no encajan en los programas de cambio conductual (Kozier, 1999).

El programa "Cuidando con amor", consideró la motivación del cambio conductual para asegurar la práctica de los conocimientos aprendidos por los familiares cuidadores. Al medir el nivel de actitud antes del programa "Cuidando con amor", se identificó que $96.7 \%$ de los participantes tenía una actitud favorable hacia la prevención de las úlceras por presión.

El concepto de actitud es complejo pues ha ido actualizándose con el transcurrir del tiempo. De todos los intentos efectuados por alcanzar una definición consensuada de este concepto, es posible que la más asumida dentro de la presente investigación sea por la de Fishbein y Ajzen (1980), definición que establece la actitud como aprendida, consistente, predisponente a la acción y evaluativa. Haciendo un consenso, la actitud se define como la predisposición para responder a un objeto particular de una manera generalmente favorable o desfavorable. La actitud viene influenciada por el propósito que se percibe, cómo se adquiere y aprende y cómo se responde al mundo. Una actitud es una expresión del presente, parte de una reflexión basada en el aprendizaje de experiencias previas. La actitud apropiada es determinante para tener la motivación apropiada para alcanzar las metas propuestas.

Pancorbo (2007), establece que el desarrollo de las úlceras por presión en un adulto mayor va unido a la formación, competencia y actitudes de las enfermeras y/o familiares cuidadores. Respecto a la actitud favorable que se obtuvo antes del programa "Cuidando con Amor", probablemente fue por el vínculo familiar de los cuidadores con los adultos mayores, pues la familia como apoyo informal se convierte en el único sustento y satisfactor de necesidades básicas, físicas, psíquicas y sociales del adulto mayor, tal como refiere CEPAL (2008), la familia funciona como un sistema formado por un conjunto de unidades interrelacionadas, con estructura y dinámica propias, con reglas y roles establecidos, donde sus integrantes interactúan con propia adaptabilidad y comunicación en factores biológicos, psicológicos y sociales.

Después del programa "Cuidando con amor" el nivel de actitud favorables sobre prevención de úlceras por presión se incrementó al 100\%. Dos aspectos relevantes que se consideran para haber obtenido este resultado, considerando que antes del programa también se tuvo un alto nivel de actitudes favorables fue en primer lugar que el $60 \%$ de los familiares cuidadores del programa "Cuidando con amor" fueron mujeres; estos datos coinciden con los reportados por Montalvo y Florez, quienes resaltan acerca de los diferentes estudios en los que referencian este rol desempeñado por la mujer (Montalvo \& Flores, 2009) y (Venegas, 2006), quien encontró en su estudio que un $85,71 \%$ son mujeres dedicadas al cuidado.

Por otro lado, en el programa "Cuidando con amor" se desarrollaron cinco sesiones de motivación para el cambio conductual: autoestima, asertividad, comunicación, resiliencia, afrontamiento del estrés, toma de decisiones y risoterapia.

El desarrollo de los talleres de motivación de cambio conductual implicó entender las fases fundamentales para el proceso de cambio que son: comprensión y desarrollo de la necesidad de cambio, es decir vender la idea y con buena y excelente publicidad de que es necesario el cambio, desarrollo de las relaciones de cambio, definir el cambio que se quiere, proceso real de moverse hacia el cambio, intento de estabilización de la situación provocada, mantenimiento, es decir, cuando se esté dando el cambio no dejarlo estático sino que hay que proceder a alimentarlo con ideas o con productos (Covey, 1996).

Para lograr el cambio fue necesario la creación de un clima propicio, basado en el respeto por las personas, lograr la participación efectiva y real, asumir una actitud experimental y curiosa, que es la parte razonable que guió a la mejor toma de decisiones, mostrar una exposición gradual a los procesos, no hacerlo en forma impulsiva, sino planeada. Utilizando estrategias como la risoterapia, talleres para afrontar el estrés y básicamente los talleres de autoestima (Covey, 1996) y (White, 1995).

Se puede concluir que los resultados positivos del nivel de actitud sobre prevención de úlceras por presión, evidencia la efectividad del programa "Cuidando con amor", porque los participantes reforzaron e incrementaron su nivel de actitudes favorables, resultados confirmados por la prueba estadística de $\mathrm{t}$ - student, con un nivel de significancia de 0.00 (Ver tabla 2).

La prácticas son habilidades o experiencias adquiridas con la realización continuada de una actividad; las conductas son comportamientos que cada individuo desarrolla en los distintos ambientes con los que se enfrenta; estas dependen de factores 
genéticos y ambientales que comienzan a ejercer su influencia desde la vida uterina y que cobran gran relevancia después del nacimiento, las conductas pueden ser instintivas y aprendidas (Gonzales, Ibarra \& Espinoza, 2007).

Conductas aprendidas o motivadas son conductas que requieren voluntad y son movidas por factores internos denominados motivos, los que nacen de las necesidades y que impulsan al hombre a la adquisición de ciertas metas. La conducta motivada es vigorosa y puede persistir por largo tiempo (Marshall, 1994).

Para el proceso del cambio intervienen la información, las actitudes y las prácticas. La información está conformada por los conocimientos que traen las personas los cuales influyen en las actitudes y estas a su vez influyen en las prácticas.

Por otro lado, los programas de intervención constituyen una clave poderosa para lograr una conducta de salud positiva (Arrivillaga, Salazar \& Correa, 2003). En otro sentido, el éxito de la prevención está en función del conocimiento, las habilidades y las actitudes de las mismas, por tanto, debemos aceptar la responsabilidad y el papel para reducir la incidencia de estas lesiones (Pancorbo 2007).

Si se analiza los cuidados en la práctica de los familiares relacionados con las medidas de prevención de las úlceras por presión, mayoritariamente se relacionan con los cuidados locales "tradicionales" asociados a las úlceras por presión, cuidados de la piel, sábanas limpias y secas, buena higiene, inspeccionar la piel y, por supuesto, los cambios posturales. Sin embargo, las intervenciones "más novedosas", utilización de superficies especiales de manejo de la presión, educación al paciente o familia, o uso de productos locales de alivio de la presión, se encuentran entre las menos conocidas. Esto, junto con el hecho de que otras recomendaciones antaño utilizadas y hoy desaconsejadas por perjudiciales (dar masajes sobre prominencias óseas, uso de dispositivos tipo rosco o el uso de piel de borrego) sigan siendo utilizadas por un número considerable de profesionales, viene a demostrar la dificultad para modificar la práctica una vez que se han interiorizado unos conocimientos, aunque estos sean variados con posterioridad (Armas, 2008).

El presente estudio evidencia el resultado que tuvo el grupo objetivo intervenido, en este caso los cuidadores en cuanto al nivel de práctica sobre prevención de úlceras por presión antes de la intervención del programa "Cuidando con amor" fue de $86.7 \%$ deficiente y $13.3 \%$ fue regular. Los resultados obtenidos antes de la intervención, son similares al estudio realizado por Solís (2007), quien encontró que la habilidad de cuidado de cuidadores de pacientes geriátricos con distintas secuelas producto de $\mathrm{ACV}$, se encontró que estos no han adquirido la habilidad de cuidado necesaria para desempeñar su rol, ubicado en la categoría media con un 51,3\%. Datos similares reportó Ramírez (2005), Venegas (2010) y Barrera et al. (2006) quienes ubican la habilidad de cuidado en categoría media, correspondiente al $41,67,37,70$ y $45,6 \%$ respectivamente. Otros estudios ubican la habilidad de cuidado en una categoría baja, como lo muestran Barrera, Pino y Sánchez (2009), en su estudio habilidad de cuidado de cuidadores familiares de personas con enfermedad crónica, una mirada internacional, en el que al comparar la habilidad de cuidado en tres países de Latinoamérica encontraron que los cuidadores tienen dificultad en la prestación del cuidado y habilidades deficientes en conocimiento, valor y paciencia.

Esta situación demanda una urgente intervención de Enfermería, para dotar a estos cuidadores de recursos y habilidades necesarias en el cuidado del anciano dependiente $\mathrm{y}$, sobre todo, realizar seguimiento y supervisión continua para garantizar un cuidado eficiente que contribuya a la calidad de vida del anciano. Aquí, se destaca la responsabilidad de los profesionales de la Enfermería no solo cuidando a este grupo, sino también, ofreciendo cursos de educación continua y capacitación al personal de salud y a los propios familiares/cuidadores del anciano. Igualmente monitorear y supervisar la realización de dichos cuidados mediante visitas domiciliarias, que garanticen el seguimiento de cada caso/anciano, a fin de disminuir la incidencia de úlceras por presión, sobre todo por el enorme costo financiero y de vida que representa su tratamiento. Asimismo, evitar masajes en las áreas de prominencias óseas por la fragilidad capilar, además de vigilar la adecuada nutrición (Venegas, 2010).

El profesional de Enfermería y los familiares cuidadores deben establecer en conjunto un plan de atención integral personalizada, reconociendo que este último permanece en mayor contacto con el anciano y será responsable de las acciones. Se concluye que los ancianos residentes en domicilio presentaron un riesgo medio a desarrollar úlceras por presión y que, aproximadamente, la mitad de ellos fue asistido por un cuidador informal o por otro familiar, entre los cuales el riesgo a úlceras por presión se clasificó como muy alto o alto respectivamente, al compararlos con aquellos cuidados por el cónyuge o hijos/ as (Venegas, 2010). 
Efectividad del programa educativo "Cuidando con amor" en los conocimientos, actitudes y prácticas sobre prevención de úlceras por presión de los cuidadores de pacientes adultos mayores del Hospital San Isidro Labrador - EsSalud, Lima, Perú

El rol de la enfermera en la prevención de la úlcera por presión e implementación del programa "cuidando con amor" fue muy importante, pues fue la encargada de plantear un plan de atención integrado y personalizado con los familiares de los pacientes que tenían riesgo de presentar UPP. La ejecución de las sesiones de capacitación de temas de prevención de UPP y los talleres de motivación personal para el cambio de conducta, donde fue necesario realizar visitas domiciliarias para completar las sesiones que no fue posible terminar durante la permanencia del paciente en el hospital; durante la visita domiciliaria se encontró que las familias realizaron cambios de conducta posterior a la ejecución del programa pues se evidenció el uso de accesorios como almohadas y otros para la prevención de UPP, además se encontró un sistema de rotación, es decir el horario de las movilizaciones del paciente, etc.

Por otro lado, después de la intervención el $100 \%$ de los participantes del programa "cuidando con amor" evidenciaron un nivel de prácticas excelente, presentando un incremento significativo. Los resultados positivos obtenidos después de la intervención en el nivel de prácticas, fueron parte de un proceso del cambio donde intervienen la información, las actitudes y las prácticas. La información está conformada por los conocimientos que traen las personas, los cuales influyen en las actitudes y estas a su vez influyen en las prácticas (Perlman \& Cosby, 1992).

Se puede concluir entonces, que el cambio positivo en el nivel de práctica al $100 \%$ excelente en los familiares cuidadores, evidencia la efectividad del programa educativo "Cuidando con amor", confirmado por la prueba de $\mathrm{t}$ - student, que indica que sí existe diferencia significativa de 0.00 , entre el nivel de prácticas de prevención de UPP antes y después del programa educativo (ver tabla 2).

Estos cambios se lograron debido a las diversas estrategias utilizadas en el programa de intervención con sesiones teóricas y prácticas referentes a la prevención de UPP con un componente motivacional, a fin de reforzar las habilidades de los familiares cuidadores para garantizar la calidad de vida del adulto mayor enfermo.

El programa "Cuidando con amor" tuvo una duración de 4 meses con la participación activa de todos los familiares, donde cada uno de ellos expresaron sus temores y sus planes en relación al cuidado del paciente con riesgo a presentar UPP, evidenciando compromiso constante con el programa, asistiendo puntualmente según los horarios establecidos; además, es necesario recalcar la participación del personal de enfermería con el programa "cuidando con amor", pues su apoyo fue vital en las sesiones de motivación de cambio conductual dirigido a los familiares, pues en cada sesión de presentación de los temas de prevención de UPP hubo temas de motivación de cambio conductual. Por otro lado, es necesario considerar la participación de las autoridades de la institución con su apoyo logístico y el permiso brindado a los familiares fuera del horario de ingreso para las capacitaciones.

Al respecto, Barrera, Pinto y Sánchez (2010), indican que los cuidadores familiares de pacientes adultos mayores en situación de cronicidad deberían ser hábiles en la prestación del cuidado, con el fin de ser asertivos en su desempeño. El cuidador que es hábil tendrá la posibilidad de encontrar significado en los hallazgos, de mantener un vínculo significativo, de hacer actividades de una forma armónica, de tomar las decisiones pertinentes para determinar el curso de la acción y poder conducir de manera honrosa su actividad; el cuidador hábil es capaz de comprender que él y la persona a su cargo son en todo momento seres trascendentes y totales que pertenecen a un contexto con el cual interactúan, que tienen una historia individual y compartida, con capacidad de desarrollarse en la acción de cuidar (Venegas, 2006).

Este estudio es útil para la profesión de enfermería, puesto que ofrece datos importantes y objetivos de quiénes y cómo son las personas que brindan cuidados a los pacientes adultos mayores de la Clínica Geriátrica San Isidro Labrador, y de esta manera continuar con este tipo de programas educativos a fin de dar una adecuada intervención en falencias que se encontraron en la habilidad de cuidado en cuanto a conocimientos, actitudes y prácticas y así ayudar a que se brinde un cuidado con calidad y mejorar el bienestar del paciente.

En conclusión el programa "Cuidando con amor" fue efectivo en incrementar el nivel de conocimientos, actitudes y prácticas sobre prevención de úlceras por presión en los cuidadores de adultos mayores del Hospital II Clínica Geriátrica San Isidro Labrador. 


\section{Declaración de financiamiento y de conflicto de intereses:}

El estudio fue financiado por la autora, quien declara no tener algún tipo de conflicto de interés en la investigación realizada.

\section{Correspondencia:}

Mg. Orfelina Arpasi Quispe

Clínica San Isidro Labrador EsSalud

Carretera Central Km 3.5 Santa Anita.

Correo electrónico: orfelina123@gmail.com.

\section{REFERENCIAS BIBLIOGRÁFICAS}

Armas Rivera, Karina L. (2008). Conocimientos que tienen los familiares sobre la prevención de úlceras por presión en pacientes con permanencia prolongada en cama en el Servicio de Medicina del HNDAC. (Tesis de maestría). Universidad Nacional Mayor de San Marcos, Lima.

Arrivillaga, M., Salazar, I., \& Correa, D. (2003). Creencias sobre la salud y su relación con las prácticas de riesgo o de protección en jóvenes universitarios. Revista Colombia Médica.

Barrera L, Blanco L, Figueroa P, Pinto N, Sánchez B. (2006). Habilidad de cuidadores familiares de personas con enfermedad crónica: una mirada internacional. Rev. Aquichan; 6 (2): 135-144.

Barrera L, Pino N, Sánchez B. (2010). Caracterización de los cuidadores familiares de América Latina. En: Cuidando a los cuidadores familiares de personas con enfermedad crónica. Grupo de Cuidado Facultad de Enfermería, Universidad Nacional de Colombia. Bogotá: Editorial Universidad Nacional de Colombia. pp. $45-56$.

Capuñay JA (2000). Presentación de enfermedades en el adulto mayor. En Varela LF: Tópicos selectos en geriatría, Lima, Universidad Peruana Cayetano Heredia.

Chávez, H. (2000). Síndromes geriátricos II. En Varela LF: Tópicos selectos en geriatría, Lima: Universidad Peruana Cayetano Heredia.

Covey, Stephen R, (2001). Hábitos de la gente altamente efectiva. Por Melton Tecnologies.

Crespo L. \& López M. (2008). Cuidadoras y cuidadores: el efecto del género en el cuidado no profesional de los mayores. Boletín sobre envejecimiento. Perfiles y Tendencias. IMSERSO; 35. 1a edición. Madrid, Ministerio de educación, política social y deporte. Disponible en: http:// www.imserso.es/InterPresent1/ groups/imserso/documents/ binario/boletinopm35.pdf.

EsSalud, (2006). "Mejoramiento de los servicios de atención geriátrica en Lima Metropolitana". Oficina central de planificación y desarrollo, Gerencia de planeamiento y evaluación de inversiones, Subgerencia de pre y post inversión, estudio de prefactibilidad del proyecto: Lima.
Fleming, K. (2008). Ultrasonido terapéutico para las úlceras de decúbito. Recuperado de: http://www.cochrane.org/ reviewa/es/ab001275.html>

Flores, Y; De La Cruz, O; Ortega, C; Hernández, S; Cabrera, F. (2010). Prevalencia de úlceras por presión en pacientes atendidos en el Instituto Nacional de Cardiología Ignacio Chávez.

Fishbein, M. \& Ajzen, I. (1980). Belief, attitude, intention, and behavior: An introduction to theory and research. Reading, MA: Addison-Wesley.

Gallo, K. \& Pachas, C. (2008). Norton en una población de ancianos de un servicio domiciliario de Lima. Programa de Prestaciones de Atención Domiciliaria/PADOMI, del Seguro Social de Salud/EsSalud; Lima - Perú.

García M, Mateo I, Maroto G. (2004). El impacto de cuidar en la salud y la calidad de vida de las mujeres. Gac Sanit,18 (Supl 2):83-92.

Gonzales, Ibarra \& Espinoza, (2007). Actitud y conocimiento del profesional de enfermería hacia el cuidado del paciente geriátrico en el Hospital Guillermo Almenara Irigoyen.

Instituto Nacional de Estadística e Informática (2007). Perfil sociodemográfico de la tercera edad. Lima Perú: Censos Nacionales 2007: XI de Población y VI de Vivienda.

Kozier, B. (1999). Fundamentos de Enfermería, conceptos, proceso y práctica. México: McGraw Hill Interamericana.

Masías J., Guillén F. \& Ribera J. (2001). Geriatría desde el principio. Barcelona: Glosa.

Marshall, R. (1994). Motivación y emoción. España: McGraw Hill Interamericana.

MINSA (2005). Guía de Implementación del Programa de Familias y Viviendas y Saludables. Dirección general de promoción de la salud: Dirección ejecutiva de promoción de vida sana; Ministerio de Salud. Lima Perú.

Montalvo A. \& Flórez I. (2009). Cuidadores de Cartagena y su contexto de cuidado: estudio comparativo. Rev. Aquichan; 9(2)135-146. 
Efectividad del programa educativo "Cuidando con amor" en los conocimientos, actitudes y prácticas sobre prevención de úlceras por presión de los cuidadores de pacientes adultos mayores del Hospital San Isidro Labrador - EsSalud, Lima, Perú

Montero, F., Arteaga, S. \& Villarreal, S. (2007). Úlceras por presión: Prevención y Tratamiento. En: Temas Prácticos en Geriatría y Gerontología. Tomo I. San José: EUNED.

Orem, Dorotea. (1980). Teoría del Autocuidado. España: Edit. Pinedo.

Organización Mundial de la Salud (2009). Medicine geriatrics and evidency -based aproach. $4^{\circ}$ edition. Estadísticas Sanitarias Mundiales.

Pancorbo, Pedro, et al. (2007). Valoración del riesgo de desarrollar úlceras por presión: uso clínico en España y metaanálisis de la efectividad de las escalas. Gerokomos v.19 n.2 Madrid.

Perlman, D., \& Cosby, C. (1992). Psicología Social. México: McGraw Hill.

Ramírez, M. (2005). Habilidad del cuidado de los cuidadores principales de paciente con cáncer gastrointestinal. Rev. Enferm; 23(2):7-17.

Rivera, H., Dávila R., González A. (2011). Calidad de vida de los cuidadores primarios de pacientes geriátricos de la Clínica de Medicina Familiar. Dr. Ignacio Chávez, ISSSTE. Rev. Esp. Med. Quir; 16(1):27-32.

Salgado, A., Guillén F. \& Ruipérez, I. (2002). Manual de Geriatría. Editorial Salvat. 3ra Edición: Barcelona: Masson (España).

Solís, M. et al. (2011). Manual de prevención y cuidados locales de heridas crónicas. Edita Servicio Cántabro de Salud; 1ra edición.
Solís, U. (2007). Síndrome de Inmovilización. En: Temas Prácticos en Geriatría y Gerontología. Tomo I. San José: EUNED.

UNESCO. (1997). Educación para la salud. Recuperado de $<$ http://www.unesco.org/education/uie/confintea/ pdf/6b_span.pdf.

Varela, L. (2009). Funcionalidad en el Adulto Mayor previa a su hospitalización a nivel nacional. Revista Médica, Instituto de Gerontología de la Universidad Cayetano Heredia 2005. Perú.

Venegas, G., Castro, J. \& Solano, M. (2010). Programa para la prevención de úlceras por presión en personas adultas mayores. Rev. Enfermería Actual en Costa Rica, No.18. recuperado de World Wide Web: http://www.revenf.ucr. ac.cr/ulceras.pdf> ISSN 1409.

Venegas B. (2006). Habilidad del cuidador y funcionalidad de la persona cuidada. Rev. Aquichan; (6):137-147.

White, E. (1995). Ministerio de curación. Biblioteca del Espíritu de Profecía. Academy Enterprises, Inc; EE. UU. de N. A.

Recibido: 09/04/2013

Aceptado: 20/09/2013 